\title{
SOME REMARKS ON SUMMABILITY FACTORS ${ }^{1}$
}

\author{
LLOYD A. GAVIN
}

ABSTRACT. Bosanquet [2] showed that a necessary and sufficient condition for $\sum_{k=1}^{\infty} x_{k} y_{k}$ to be Cesàro summable of order $n$ ( $n$ is a nonnegative integer) whenever $\sigma_{k}^{n}(y)=O(k)$ where $\sigma_{k}^{n}(y)$ is the $k$ th Cesàro mean of $y$ of order $n$ is that $\sum_{k=1}^{\infty} k^{n+1}\left|\Delta^{n+1} x_{k}\right|<\infty$ and $\lim _{k \rightarrow 0} k x_{k}=0$. The main result of this paper is to show that a necessary and sufficient condition for $\sum_{k=1}^{\infty} x_{k} y_{k}$ to be Cesàro summable of order $n$ ( $n$ is a nonnegative integer) whenever $\sum_{k=1}^{\infty} k^{n+1}\left|\Delta^{n+1} x_{k}\right|<\infty$ and $\lim _{k \rightarrow \infty} k x_{k}=0$ is that $\sigma_{k}^{n}(y)=O(k)$.

Introduction. Let the linear space of all complex sequences be denoted by $\omega$. Any linear subspace of $\omega$ is called a sequence space. A sequence space which is a Banach space such that for $k=1,2, \ldots$ the linear functionals $f_{k}$, where $f_{k}(x)=x_{k}$ are continuous, is called a BK-space. Let $m$ be a nonnegative integer, and $E$ a Banach space containing $e_{n}=\left\{\delta_{n k}\right\}_{k=1}^{\infty}, n=1,2, \ldots$ If each $x \in E$ has the property that

$$
\sigma_{n}^{m}(x)=\sum_{k=1}^{n} \frac{1}{\left(\begin{array}{c}
m+n \\
n
\end{array}\right)}\left(\begin{array}{c}
m+n-k \\
m
\end{array}\right) x_{k} e_{k} \in E \text { for } n=1,2, \ldots
$$

and $x=\lim _{n \rightarrow \infty} \sigma_{n}^{m}(x)$, then $E$ is called a BK-space with $(C, m)-A K$. It is well known that $(C, m)-A K$ implies $(C, m+k)-A K$ for $k=0,1,2, \ldots$ and $m=0,1,2, \ldots$.

Let $E$ and $F$ be sequence spaces. $(E \rightarrow F)=\left\{x \in \omega: x y=\left\{x_{k} y_{k}\right\}_{k=1}^{\infty} \in F\right.$ for all $y \in E$ \} is called the space of multipliers from $E$ into $F$. Associated with each sequence space $E$ are the integrated and differentiated spaces of $E$ defined respectively by

$$
\begin{gathered}
\int E=\left\{x \in \omega: d x=\left\{k x_{k}\right\}_{k=1}^{\infty} \in E\right\}, \\
d E=\left\{x \in \omega: \int x=\left\{\frac{x_{k}}{k}\right\}_{k=1}^{\infty} \in E\right\} .
\end{gathered}
$$

The following sequence spaces will be considered.

Received by the editors October 8, 1974.

AMS (MOS) subject classifications (1970). Primary 40D15.

Key words and phrases. Summability factors.

${ }^{1}$ This work is partially excerpted from the author's Ph.D. thesis written at Illinois Institute of Technology under Professor Gunther Goes. 


$$
\begin{aligned}
& l^{\infty}=\left\{x \in \omega: \sup _{k}\left|x_{k}\right|<\infty\right\}, \\
& l=\left\{x \in \omega: \sum_{k=1}^{\infty}\left|x_{k}\right|<\infty\right\}, \\
& c_{0}=\left\{x \in \omega: \lim _{k \rightarrow \infty} x_{k}=0\right\}, \\
& b v_{0}=\left\{x \in \omega: \sum_{k=1}^{\infty}\left|x_{k}-x_{k+1}\right|<\infty \text { and } \lim _{k \rightarrow \infty} x_{k}=0\right\}, \\
& q_{0}=\left\{x \in \omega: \sum_{k=1}^{\infty}(k+1)\left|\Delta^{2} x_{k}\right|<\infty \text { and } \lim _{k \rightarrow \infty} x_{k}=0\right\}, \\
& \sigma_{\infty}=\left\{x \in \omega: \sup _{k}\left|k^{-1} \sum_{n=1}^{k} x_{n}\right|<\infty\right\} .
\end{aligned}
$$

For $x \in \omega$, set

$$
\begin{aligned}
& s_{n}^{0}(x)=x_{1}+x_{2}+\cdots+x_{n}, \\
& s_{n}^{1}(x)=s_{1}^{0}(x)+s_{2}^{0}(x)+\cdots+s_{n}^{0}(x), \\
& \quad \vdots \\
& s_{n}^{k}(x)=s_{1}^{k-1}(x)+s_{2}^{k-1}(x)+\cdots+s_{n}^{k-1}(x) ; k, n=1,2,3, \ldots
\end{aligned}
$$

Set $\quad \sigma_{n}^{k}(x)=s_{n}^{k}(x) /\left(\begin{array}{c}n+k \\ k\end{array}\right), \quad k=0,1,2, \ldots$ and $n=1,2, \ldots \quad \sigma_{n}^{k}(x) \quad$ is called the $n$th Cesàro mean of $x$ of order $k$. If $\lim _{n \rightarrow \infty} \sigma_{n}^{k}(x)=s$, we say $\sum_{n=1}^{\infty} x_{n}$ is $(C, k)$ summable and denote this by $(C, k)-\sum_{n=1}^{\infty} x_{n}=s$.

For $k=0,1,2, \cdots$ define

$C_{k}=\left\{x \in \omega: \lim _{n \rightarrow \infty} \sigma_{n}^{k}(x)\right.$ exists $\}$,

${ }^{\prime} C_{k}=\left\{x \in \omega: \sup _{n}\left|\sigma_{n}^{k}(x)\right|<\infty\right\}$, and

$\sigma_{k, \infty}=\left\{x \in \omega: \sup _{n}\left|n^{-1} \sigma_{n}^{k}(x)\right|<\infty\right\}$.

$h_{b}^{k+1}=\left\{x \in \omega: \sum_{n=1}^{\infty} n^{k+1}\left|\Delta^{k+1} x_{n}\right|<\infty\right\}$ and $h^{k+1}=h_{b}^{k+1} \cap \int c_{0}$ where

$\Delta^{1} x_{\eta}=x_{n}-x_{n+1}$ and $\Delta^{k+1} x_{n}=\Delta^{1}\left(\Delta^{k} x_{n}\right), k=1,2,3, \ldots$,

$v^{k+1}=\left\{x \in \omega: \sum_{n=1}^{\infty}(n+1)^{k}\left|\Delta^{k+1} x_{n}\right|<\infty\right\}$, and

$v_{0}^{k+1}=v^{k+1} \cap c_{0}$.

Let $E$ be a sequence space and $n$ a nonnegative integer. The set of all $y \in \omega$ such that $(C, n)-\sum_{k=1}^{\infty} x_{k} y_{k}$ exists for all $x \in E$ is called the set of summability factors of $E$ of order $n$. Clearly the set of summability factors of $E$ of order $n$ is the set of multipliers $\left(E \rightarrow C_{n}\right)$. Using the multiplier notation, Bosanquet [2, p. 296, Theorem A] showed that $\left(\sigma_{n, \infty} \rightarrow C_{n}\right)=h^{n+1}$ for $n=0,1,2, \ldots$ Our main result stated in multiplier notation is $\left(h^{n+1} \rightarrow C_{n}\right)$ $=\sigma_{n, \infty}$ for $n=0,1,2, \ldots$ (See Theorem 2.12.)

\section{Preliminary statements.}

1.1 Proposition. For $m=1,2, \ldots, h^{m+1} \subset h^{m}$.

Proof. Let $m$ be a positive integer and let $x \in h^{m+1}$. Then $\sum_{k=1}^{\infty} k^{m+1}\left|\Delta^{m+1} x_{k}\right|<\infty$ and $\lim _{k \rightarrow \infty} x_{k}=0$. Set $\varepsilon_{k}=\Delta^{m} x_{k}$; then $\Delta \varepsilon_{k}$ $=\Delta^{m+1} x_{k}$. Hence $\sum_{k=1}^{\infty} k^{m+1}\left|\Delta \varepsilon_{k}\right|=\sum_{k=1}^{\infty} k^{m+1}\left|\Delta^{m+1} x_{k}\right|<\infty$. By [1, p. 42, Lemma 6] there exists a number $s$ such that

(i) $\varepsilon_{k}=s+o\left(1 / k^{m+1}\right)$ and

(ii) $\sum_{k=1}^{\infty} k^{m}\left|\varepsilon_{k}-s\right|<\infty$.

Since $h^{m} \subset c_{0}$,

$$
\lim _{k \rightarrow \infty} \varepsilon_{k}=\lim _{k \rightarrow \infty} \Delta^{m} x_{k}=0 .
$$

By (1.2) and (i), $s=0$. By (ii) $\sum_{k=1}^{\infty} k^{m}\left|\Delta^{m} x_{k}\right|<\infty$. Hence $x \in h^{m}$. 
Remark. By [5, p. 96, Theorem 3.2], $h^{1} \subset l$. Hence $h^{m} \subset l$ for $m=1,2,3$, .... (See 1.1.)

The following results will be used:

1.3. If $E$ and $F$ are BK-spaces with $(C, k)-A K$, then $\left(E \cap F \rightarrow C_{k}\right)$ $=\left(E \rightarrow C_{k}\right)+\left(F \rightarrow C_{k}\right)[4$, p. 156, Theorem 4].

1.4. If $r>-1, p \geqslant 0, \sum_{k=1}^{\infty} k^{p-1}\left|x_{k}\right|<\infty$, then $\sum_{k=1}^{\infty} k^{p+r}\left|\Delta^{r+1} x_{k}\right|$ $<\infty$ if and only if $\sum_{k=1}^{\infty} k^{p+r+1}\left|\Delta^{r+1}\left(x_{k} / k\right)\right|<\infty[3$, p. 77].

If $p=0$ in 1.4, we get for $r=1,2, \ldots, d l \cap d h_{b}^{r+1}=d l \cap v^{r+1}$. Hence for $r=1,2, \ldots, l \cap h_{b}^{r+1}=l \cap \int v^{r+1}$. Since $h^{r+1} \subset l$ for $r=0,1,2$,

$$
h^{r+1}=\int v_{0}^{r+1} \cap l .
$$

\section{The space of multipliers from $h^{n}$ into $C_{n-1}$.}

2.1 Lemma. $l^{\infty} \subset \sigma_{n-1, \infty}, \quad n=1,2, \ldots$

PRoof. The proof is by induction. Since $\sigma_{0, \infty}=\sigma_{\infty}$ the statement is true for $n=1$. Assume

$$
\begin{aligned}
l^{\infty} & \subset \sigma_{k, \infty} \quad \text { for } 1 \leqslant k<n, \\
\left|\frac{s_{r}^{n}(x)}{r^{n+1}}\right| & <\frac{1}{r} \sum_{j=1}^{r}\left|\frac{s_{j}^{n-1}(x)}{j^{n}}\right| .
\end{aligned}
$$

If $x \in l^{\infty}$, then by (2.2) $\sup _{j}\left|s_{j}^{n-1}(x) / j^{n}\right| \leqslant M<\infty$. Hence (2.3) implies $\sup _{r}\left|s_{r}^{n}(x) / r^{n+1}\right| \leqslant M$. Thus $l^{\infty} \subset \sigma_{n, \infty}$.

2.4 Lemma. For $k=0,1,2, \ldots, n=1,2, \ldots$ and $x \in \omega$,

$$
s_{n}^{k}(d x)=n s_{n}^{k}(x)-(k+1) s_{n-1}^{k+1}(x) .
$$

Proof. Follows immediately from Abel's partial summation and definition of $s_{n}^{k}(x)$.

2.5 Proposition. For $k=0,1,2, \ldots, d^{\prime} C_{k} \subset \sigma_{k, \infty}$.

Proof. It suffices to show that ' $C_{k} \subset \int \sigma_{k, \infty}$ for $k=0,1,2, \ldots$ Let $x \in \in^{\prime} C_{k}$, then

$$
\sup _{n}\left|\frac{s^{k}(x)}{n^{k}}\right|=M<\infty \text {. }
$$

Since ' $C_{k} \subset{ }^{\prime} C_{k+1}$ for $k=0,1,2, \ldots$,

$$
\sup _{n}\left|\frac{s_{n}^{k+1}(x)}{n^{k+1}}\right|=M^{\prime}<\infty
$$

for some $M^{\prime}>0$. From 2.4 we obtain

$$
s_{n}^{k}(d x)=n s_{n}^{k}(x)-(k+1) s_{n-1}^{k+1}(x) \text { for } n=1,2, \ldots
$$

Now (2.6), (2.7) and (2.8) imply 


$$
\left|\frac{s_{n}^{k}(d x)}{n^{k+1}}\right| \leqslant\left|\frac{s_{n}^{k}(x)}{n^{k}}\right|+(k+1)\left|\frac{s_{n-1}^{k+1}(x)}{n^{k+1}}\right| \leqslant M+(k+1) M^{\prime}
$$

for all $n$. Hence $d x \in \sigma_{k, \infty}$, i.e., $x \in \int \sigma_{k, \infty}$.

2.9 TheOREM. For $n=0,1,2, \ldots, \quad \sigma_{n, \infty}=\left(\left(\sigma_{n, \infty} \rightarrow C_{n}\right) \rightarrow C_{n}\right)$.

Proof. Evidently $\sigma_{n, \infty} \subseteq\left(\left(\sigma_{n, \infty} \rightarrow C_{n}\right) \rightarrow C_{n}\right)$ for all $n$. Bosanquet proved that $\left(\sigma_{n, \infty} \rightarrow C_{n}\right)=h^{n+1}$ for $n=0,1,2, \ldots[2$, p. 296, Theorem A]. For $n=0,1,2, \ldots, h^{n+1}=\int v_{0}^{n+1} \cap l, n=0,1,2, \ldots$ By $[4$, p. 155, Theorem 3], $\int v_{0}^{n+1}$ is a BK-space with $(C, n)-A K$. Since $l$ is a BK-space with $A K$, it also has $(C, n)-A K$. From [4, p. 165, Theorem 4]

$$
\begin{aligned}
\left(\left(\sigma_{n, \infty} \rightarrow C_{n}\right) \rightarrow C_{n}\right) & =\left(\int v_{0}^{n+1} \cap l \rightarrow C_{n}\right) \\
& =\left(\int v_{0}^{n+1} \rightarrow C_{n}\right)+\left(l \rightarrow C_{n}\right) \text { for } n=0,1,2, \ldots
\end{aligned}
$$

$\left(\int v_{0}^{n+1} \rightarrow C_{n}\right)=d^{\prime} C_{n}, n=0,1,2, \ldots[4$, p. 156, Theorem 5]. Since $l$ has $A K$, $\left(l \rightarrow C_{n}\right)=\left(l \rightarrow C_{0}\right)=l^{\infty}$. Thus, by 2.1 and 2.5 ,

$$
\left(\left(\sigma_{n, \infty} \rightarrow C_{n}\right) \rightarrow C_{n}\right)=d^{\prime} C_{n}+l^{\infty} \subset \sigma_{n, \infty} .
$$

Hence $\left(\left(\sigma_{n, \infty} \rightarrow C_{n}\right) \rightarrow C_{n}\right)=\sigma_{n, \infty}$ for $n=0,1,2, \ldots$

2.11 Corollary. For $n=0,1,2, \ldots, \sigma_{n, \infty}=d^{\prime} C_{n}+l^{\infty}$.

Proof. See (2.10) in proof of 2.9 .

Our main result can now be stated and proved.

2.12 Theorem. For $n=1,2, \ldots,\left(h^{n} \rightarrow C_{n-1}\right)=\sigma_{n-1, \infty}$.

Proof. By $2.9 \sigma_{n, \infty}=\left(\left(\sigma_{n, \infty} \rightarrow C_{n}\right) \rightarrow C_{n}\right)$ for $n=0,1,2, \ldots$,

$$
h^{n}=\left(\sigma_{n-1, \infty} \rightarrow C_{n-1}\right) \text { for } n=1,2, \ldots
$$

[2, p. 296, Theorem A]. Hence

$$
\left(h^{n} \rightarrow C_{n-1}\right)=\left(\left(\sigma_{n-1, \infty} \rightarrow C_{n-1}\right) \rightarrow C_{n-1}\right) \text { for } n=1,2, \ldots
$$

2.13 Corollary. For $n=1,2, \ldots,\left(\left(h^{n} \rightarrow C_{n-1}\right) \rightarrow C_{n-1}\right)=h^{n}$.

Proof. Let $n$ be a positive integer. $\left(h^{n} \rightarrow C_{n-1}\right)=\sigma_{n-1, \infty}$ by 2.12 .

$$
\left(\sigma_{n-1, \infty} \rightarrow C_{n-1}\right)=h^{n}
$$

by $[2$, p. 296, Theorem A].

\section{REFERENCES}

1. L. S. Bosanquet, Note on convergence and summability factors, J. London Math. Soc. 20 (1945), 39-48. MR 7, 432.

2. - Note on convergence and summability factors. II, Proc. London Math. Soc. (2) 50 (1948), 295-304. MR 10, 112. 
3. L. S. Bosanquet and H. C. Chow, Some remarks on convergence and summability factors, J. London Math. Soc. 32 (1957). 73-82. MR 18, 733.

4. G. Goes, Bounded variation sequences of order $k$ and representation of null sequences, J. Reine Angew. Math. 253 (1972),152-161. MR 45 \# 9027.

5. G. Goes and S. Goes, Sequences of bounded variation and sequences of Fourier coefficients. I, Math. Z. 118 (1970), 93-102.

Department of Mathematics, California State University, SaCramento, California 95819 (Current address)

Department of Mathematics, Xavier University of Louisiana, New Orleans, Louisiana 70125 